\title{
Das biomechanische Konzept der zementfreien anisotropen Pfanne "Vektor" in der Hüftendoprothetik
}

\section{Biomechanical concept of the cementless anisotropic cup "Vektor" in total hip arthroplasty}

\begin{abstract}
In der Hüftendoprothetik können Relativbewegungen, die an der Kontaktfläche Pfanne/Acetabulum auftreten und durch unterschiedliche Steifigkeiten des knöchernen Lagers und des Pfannenimplantates hervorgerufen werden zu Knochenabbau und Implantatlockerungen führen. Da sich der Elastizitätsmodul des Knochens aufgrund von strukturellen Entwicklungen kontinuierlich ändert, somit Isoelastizität nie erreicht werden kann, wurde die anisotrope "Vektorpfanne" entwickelt. Ziel der Arbeit ist die Überprüfung der Hypothese, dass tatsächlich ein anisotropes Verhalten des Implantates vorliegt.

Die anisotrope Vektorpfanne ist sphärisch, sie besteht aus der Titanlegierung TiAl5Fe2,5. Der metallische Pfannenträger ist im cranialen Bereich starr und dickwandig ausgelegt, während die caudale Seite eine definierte Flexibilität durch eine offene dünnwandige Rippenkonstruktion aufweist. Es wurden Elastizitätsmessungen unterschiedlicher Implantate an der Prüfmaschine MTS 810 durchgeführt und das Federverhaltens der anisotropen Flügel im elastischen Bereich überprüft.

Die Messungen belegen, dass das Konzept der Anisotropie verwirklicht werden konnte, die äquatoriale Einsägung reduziert die Federkonstante auf 1/8.
\end{abstract}

Alexander Schuh', Miroslav Karan ${ }^{1}$, Ulrich Holzwarth ${ }^{2}$, Roger Thull', Günther Zeiler ${ }^{1}$

\section{Schlagworte:}

Anisotropie - Vektorpfanne - zementfrei Federkonstante - Hüftendoprothetik

\section{Keywords:}

Vektorcup - cementless cup- spring rate total hip replacement

\section{Einleitung}

Der Begriff "Anisotropie“ bezeichnet in der Naturwissenschaft die Richtungsabhängigkeit physikalischer Eigenschaften eines Materials. Nach dem Hooke'schen Gesetz bedingen auf einen Körper einwirkende Kräfte immer dessen Verformung. Der Umfang der Verformung hängt in hohem Maße von der Qualität des verformten Materials, seinen Formen und Dimensionen und von der Größe der einwirkenden Kraft $\mathrm{ab}$. In der Hüftendoprothetik müssen alle Pfannenimplantate ein unterschiedliches Deformationsverhalten des knöchernen Acetabulums und der Pfannenimplantate unter Belastung auslösen, weil isoelastische Implantate derzeit nicht realisierbar sind [3]. Der Grund hierfür sind Veränderungen der mechanischen Qualität des Knochenlagers im Laufe des Lebens eines Individuums und im Rahmen der Ausprägung einer Hüftgelenksarthrose mit der Ausbildung von Strukturdefekten und der Reduktion der Knochendichte. Das natürliche Acetabulum weist in seiner intakten Form die höchste Substanzdichte im kranialen Pfannenabschnitt als leicht

I Orthopädische Klinik Rummelsberg, Schwarzenbruck

2 Peter Brehm, Chirurgic Mechanik, Weisendorf.

3 Lehrstuhl für Funktionswerkstoffe in Medizin und Zahnheilkunde, Wurzburg asphärische, subchondrale Sklerosezone auf. Über ihr liegt ein spongiöser Knochenverbund, der zusammen mit seiner Flüssigkeitsfüllung die knorpelnahe Sklerosa abstützt und sie auch bei Spitzenbelastung nur eine geringgradige Deformation erfahren lässt $[1,2]$.

Die Masse der knöchernen Substanz nimmt hin zu den Facies lunatae ab und findet in der Incisura acetabuli eine Minimierung der schalenartigen Strukturanordnung um das Acetabulum. Die Mehrzahl der menschlichen Acetabula weisen cranial eine elliptoide Form auf. Selten gibt es auch näherungsweise sphärische Acetabula. Die Verformung des knöchernen Acetabulums ist auch von der Qualität des Knochens und den bestehenden Knochendefekten abhängig. Die Erhaltung der subchondralen Sklerosa ist für die Aufnahme der regulären kranialen Druckbelastungen von entscheidender Bedeutung. Diese Lasten sind dreimal so groß wie die Belastungsstabilität einer gut strukturierten Spongiosa [5, 6, 7]. Der Knochen ist als ein anisotroper Werkstoff anzusehen. Seine Belastungsstabilitäten liegen bei der Spongiosa je nach Strukturdichte und Mineralisationsgrad zwischen 1,5 und $2,5 \mathrm{~N} / \mathrm{mm}^{2}$ und bei der Kortikalis zwischen 5,0 und $10,0 \mathrm{~N} / \mathrm{mm}^{2}$. Bei normalgewichtigen Menschen beträgt die Belastung der kranialen Zirkumferenz des Acetabulums 6,0 bis $10,0 \mathrm{~N} / \mathrm{mm}^{2}$. 


\section{Material und Methode}

Die anisotrope Vektorpfanne ist sphärisch, sie besteht aus der Titanlegierung TiAl5Fe2,5. Der metallische Pfannenträger ist im kranialen Bereich starr und dickwandig ausgelegt, während die caudale Seite eine definierte Flexibilität durch eine offene dünnwandige Rippenkonstruktion aufweist. Ihre Außendurchmesser stehen in $2 \mathrm{~mm}$-Schritten zwischen 44 und $60 \mathrm{~mm}$ zur Verfügung. Die Oberfläche ist edelkorundgestrahlt mit einer Rauheit von $60 \mu \mathrm{m}$ (Abb. 1).

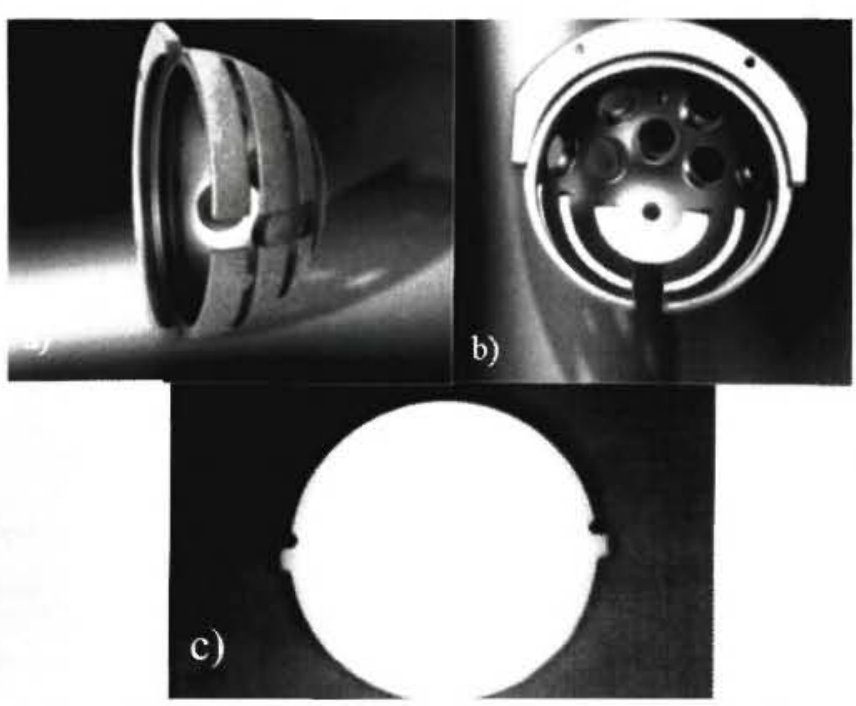

Abb. 1: a) Metallischer Teil der anisotropen Vektorpfanne in der Sicht von caudal. Die spangenartige Konstruktion ermöglicht eine zunehmende Elastizität nach caudal. Die Außenfläche ist rau $(60 \mu \mathrm{m})$ gestrahlt.

b) Für die Schraubenfixation werden im Regelfall die zentralen drei Bohrungen verwendet. Die randständigen Bohröffnungen kommen bei ausgeprägten Defekten, Revisionseingriffen und großen Pfannendachplastiken zum Einsatz. Die Innenseite der Pfanne ist glatt gestrahlt $(10 \mu \mathrm{m})$.

c) Der Polyethyleneinsatz überragt die Titanschale randständig etwas, ist mit einem zirkulär auf der Außenseite angebrachten Klemmring versehen und weist zwei Rotationssicherungen auf.

Je ein Exemplar der zementfreien anisotropen Vektorpfanne mit einem Durchmesser von $52 \mathrm{~mm}$ und $60 \mathrm{~mm}$ wurden auf einer servohydraulischen Prüfmaschine Typ MTS 810 mit separater Regelung für statisches und dynamisches Testen (MTS, Minneapolis, Minnesota) im elastischen Bereich statisch auf das Federverhalten der Pfannenspangen geprüft (Abb. 2).

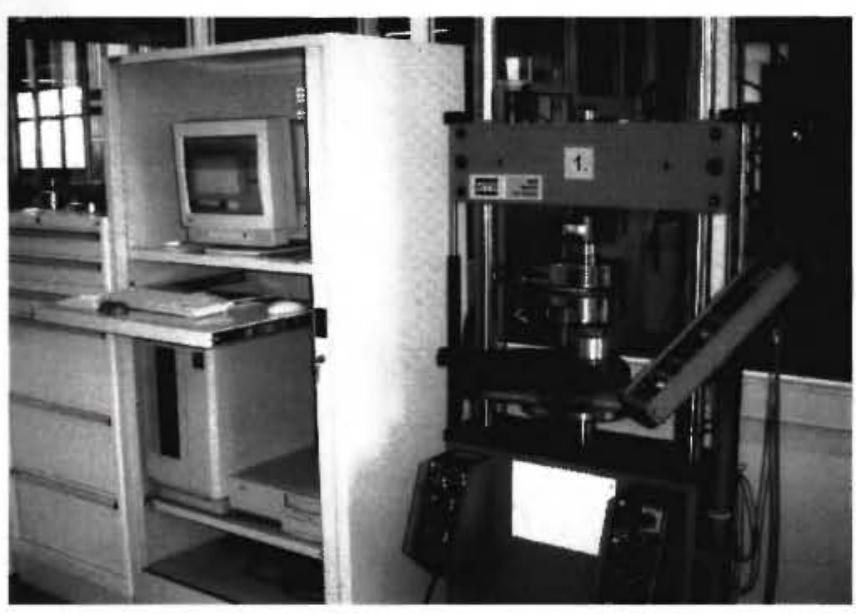

Abb. 2: Prüfmaschine Typ MTS 810 (MTS, Minneapolis, Minnesota)
Alle Messungen wurden jeweils 3 Mal durchgeführt und der Mittelwert berechnet.

Die mechanisch-physikalische Messgröße ist die Bestimmung der Federrate gemäß der Gleichung

$\mathrm{c}=\mathrm{s} / \mathrm{F}$

(mit $\mathrm{c}=$ Federrate $[\mathrm{mm} / \mathrm{N}], \mathrm{s}=$ Weg $[\mathrm{mm}]$ und $\mathrm{F}=\operatorname{Kraft}[\mathrm{N}]$ ).

Im elastischen Bereich ergibt sich aus der Aufzeichnung des Verformungs- (Weg) und Kraftabschnittes die Federrate. Die Federrate c kann gemäß obiger Gleichung aus dem KraftWeg-Diagramm als Steigung ermittelt werden.

Die Messergebnisse wurden in eine Excel-Datei übertragen, die Kraft-Weg-Kurve und die Steigung im elastischen Bereich berechnet.

In Abhängigkeit von Elastizitätsmodul des Werkstoffs und Geometrie der Einfräsung sowie der Wandstärke stellt sich die Federrate als Funktion der mehr oder weniger steifen Konstruktion des Bauteils dar. Es wurde jeweils eine Messung am Ursprung der Spangen der $52 \mathrm{~mm}$ und $60 \mathrm{~mm}$ Vektorpfanne durchgeführt, um die dem Bauteil eigene nicht durch die Spangenkonstruktion bedingte Federrate zu messen.

Neben der Prüfung einer einzelnen Spange des Implantates wurde auch die Federrate von beiden Spangen einer Pfanne gemessen. Rein theoretisch sollte hier eine um 50\% höhere Federrate als Ergebnis darstellbar sein, da beide Spangen aus dem identischen Werkstoff bestehen und dieselbe Geometrie aufweisen (Abb. 3).
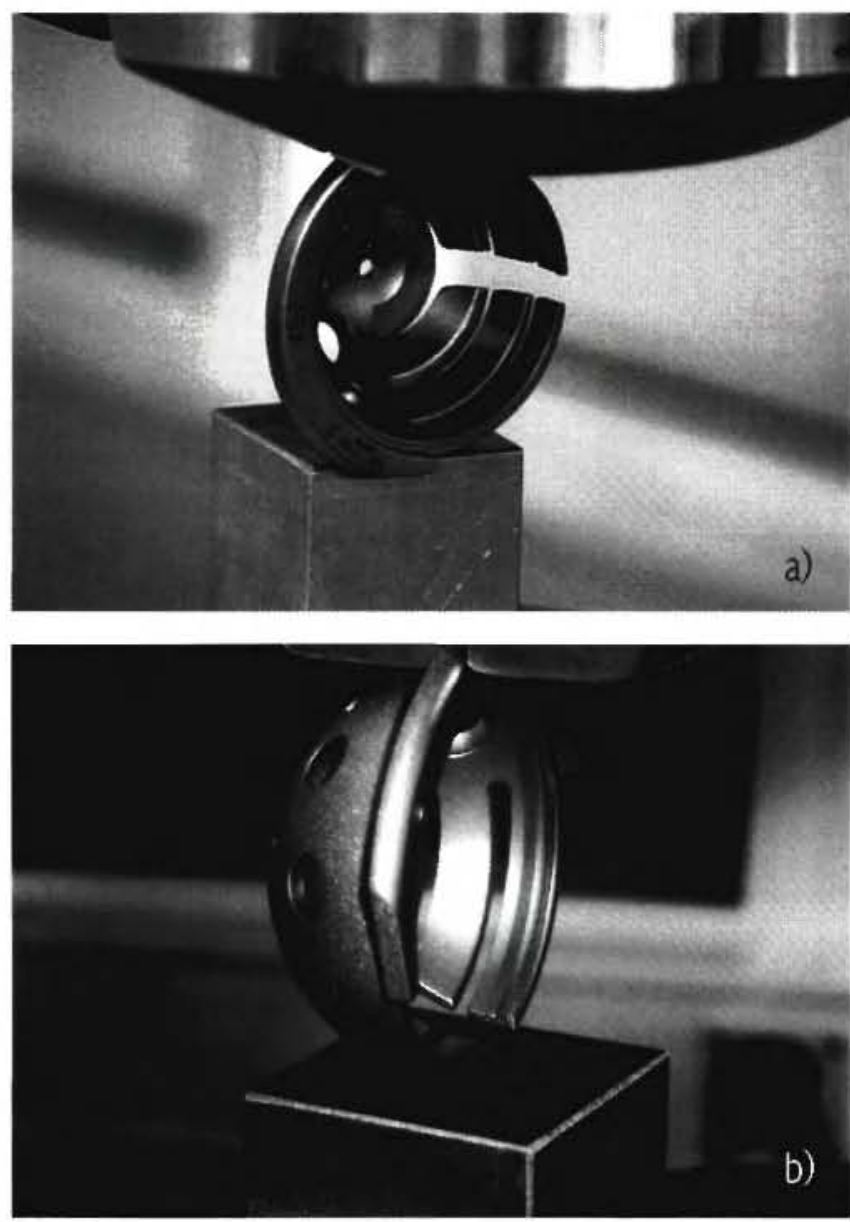

Abb. 3:

a) Messung des E-Moduls am Ursprung der caudalen Spange

b) Messung des E-Moduls am Ende der caudalen Spange 

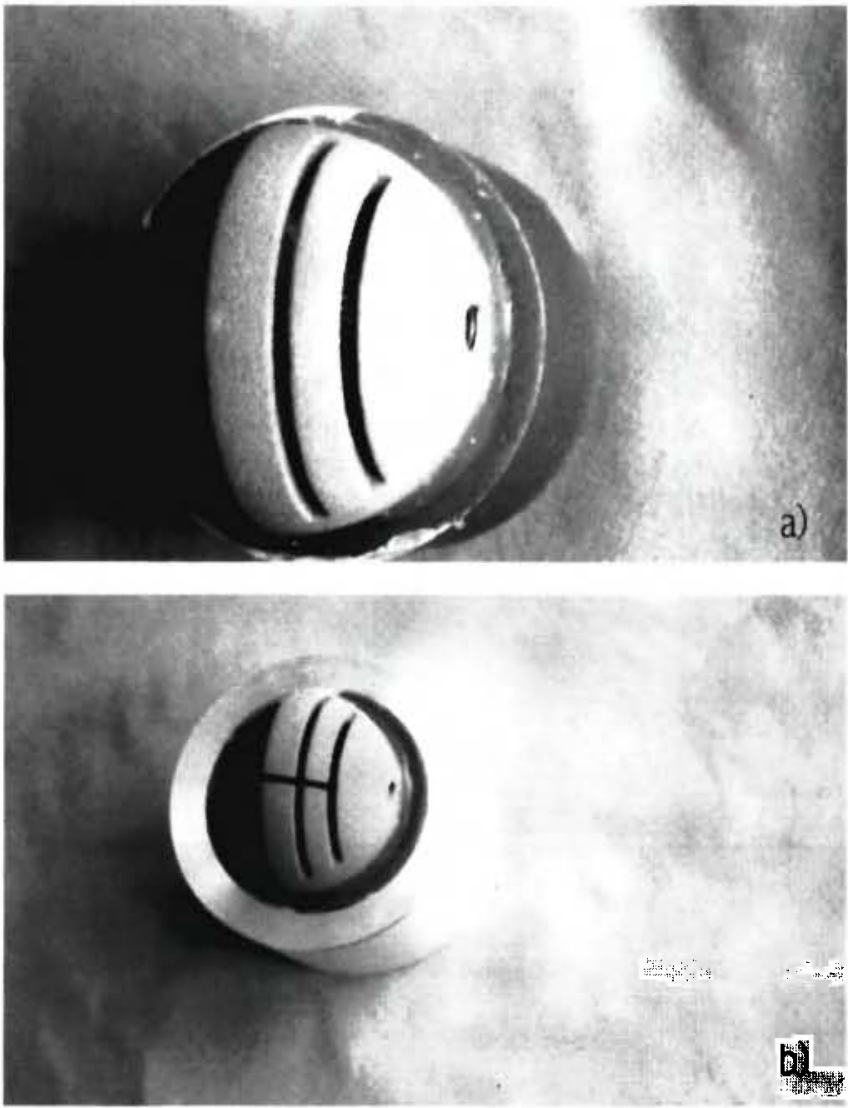

Um den Elastizitätseffekt der Einfräsung vom Äquator zum Pol zu prüfen, wurde auch an einem Pfannenprototyp vor Einfräsung die dem Bauteil eigene Federrate und nach Einfräsung die Federrate der entstandenen rechten und linken caudalen Spange gemessen (Abb. 4).

\section{Ergebnisse}

Die Federkostante der $60 \mathrm{~mm}$ Pfanne am Lamellenursprung betrug $970 \mathrm{~mm} / \mathrm{N}$, der einzelnen Spange $74 \mathrm{~mm} / \mathrm{N}$ und beider Spangen $123 \mathrm{~mm} / \mathrm{N}$. Für die $52 \mathrm{~mm}$ Pfanne betrugen die Werte am Lamellenursprung $1098 \mathrm{~mm} / \mathrm{N}$, der einzelnen Spange $91 \mathrm{~mm} / \mathrm{N}$ und beider Spangen $176 \mathrm{~mm} / \mathrm{N}$. Bei dem nicht eingesägten Prototyp fanden wir $1870 \mathrm{~mm} / \mathrm{N}$, nach der äquatorialen Einsägung $235 \mathrm{~mm} / \mathrm{N}$ (Tab. 1).

In Abbildung 5 ist beispielhaft die Kraft- Weg- Kurve des Prototyps dargestellt. Durch die äquatoriale Einsägung wird dich Federrate auf $1 / 8$ reduziert.

\begin{tabular}{|c|c|c|c|c|c|}
\hline & $\begin{array}{l}\text { rechte } \\
\text { Spange }\end{array}$ & $\begin{array}{l}\text { linke } \\
\text { Spange }\end{array}$ & $\begin{array}{l}\text { beide } \\
\text { Spangen }\end{array}$ & Spangenursprung & $\begin{array}{l}\text { Vor } \\
\text { Einsägung }\end{array}$ \\
\hline $\begin{array}{l}\text { 60er } \\
\text { Pfanne }\end{array}$ & $\begin{array}{l}78.46 \\
\mathrm{~N} / \mathrm{mm}\end{array}$ & $\begin{array}{l}74.42 \\
\mathrm{~N} / \mathrm{mm}\end{array}$ & $\begin{array}{l}123,14 \\
\mathrm{~N} / \mathrm{mm}\end{array}$ & $970,05 \mathrm{~N} / \mathrm{mm}$ & -- \\
\hline $\begin{array}{l}\text { 52er } \\
\text { Pfanne }\end{array}$ & $\begin{array}{l}91,47 \\
\mathrm{~N} / \mathrm{mm}\end{array}$ & $\begin{array}{l}109,39 \\
\mathrm{~N} / \mathrm{mm}\end{array}$ & $\begin{array}{l}176.00 \\
\mathrm{~N} / \mathrm{mm}\end{array}$ & $1097.78 \mathrm{~N} / \mathrm{mm}$ & -- \\
\hline Prototyp & $\begin{array}{l}299.94 \\
\mathrm{~N} / \mathrm{mm}\end{array}$ & $\begin{array}{l}211.92 \\
\mathrm{~N} / \mathrm{mm}\end{array}$ & & & $\begin{array}{l}1851.28 \\
\mathrm{~N} / \mathrm{mm}\end{array}$ \\
\hline
\end{tabular}

Tab. l: Federraten in $\mathrm{N} / \mathrm{mm}$

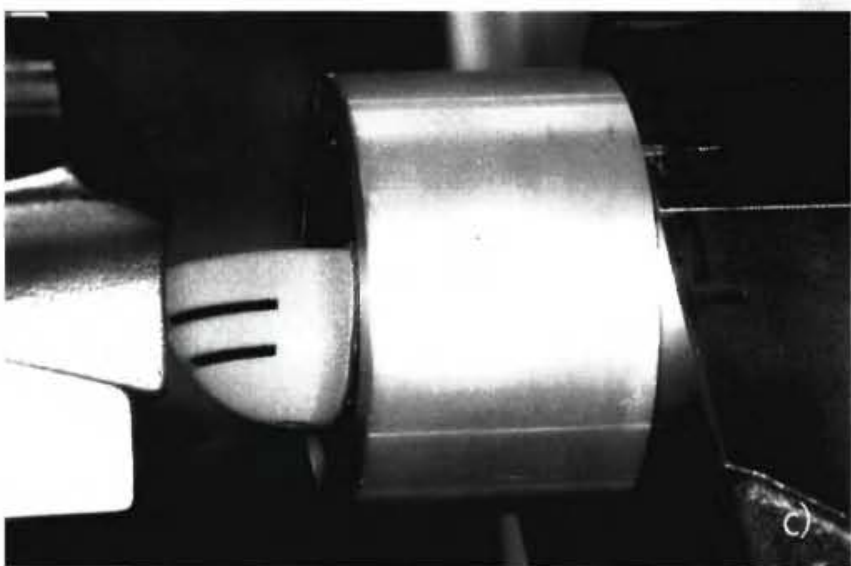

Abb. 4: In PMMA- Zement eingebetteter Prototyp

a) Vor äquatorialer Einsägung

b) Nach äquatorialer Einsägung

c) Im Prürstand zur Messung des E-Moduls des Prototyps

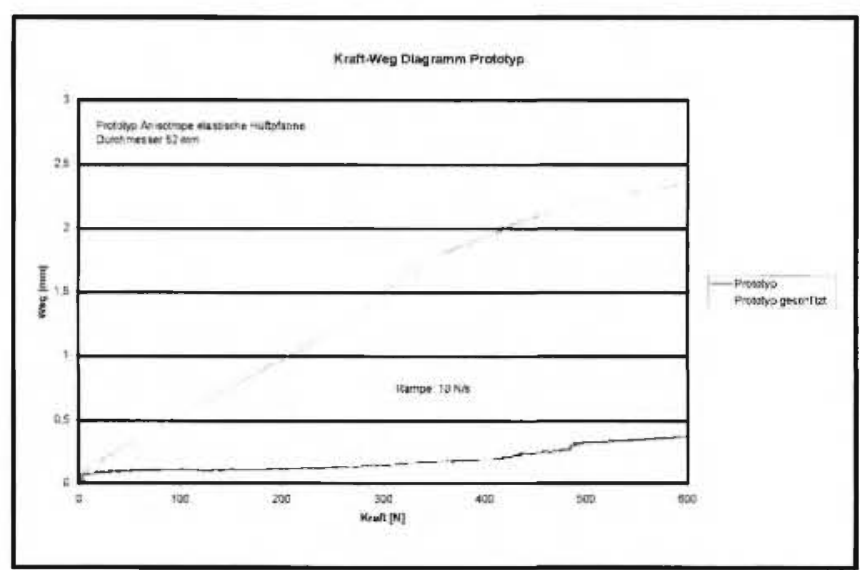

Abbildung 5: Kraft-Weg- Diagramm des Prototyps

\section{Diskussion}

Die Philosophie der anisotropen Vektorpfanne zielt auf die Erhaltung aller für die Pfannenverankerung geeigneter knöcherner Strukturen des Acetabulums, insbesondere seiner subchondralen Kompakta. Dieser Philosophie kann nur ein sphärisches Pfannenimplantat gerecht werden [4]. Aus gleichem Grunde wurde auch auf die Entwicklung gewindeartiger Verankerungsstrukturen verzichtet $[1,3,6]$. Jede Abnahme der Wandstärke des Acetabulums führt unter Lastfluss zu einer stärkeren Verformung des Acetabulums.

In Laboruntersuchungen an Beckenknochen konnte nachgewiesen werden, dass die bei gleichen Kraftflüssen resultierenden Verformungen des Acetabulums im unbehandelten Zustand gegenüber denen einer sparsamen sphärischen Fräsung mit Implantation der anisotropen Vektorpfanne die geringste Veränderung nach der Implantation erfuhren [8]. Eine Deformation der Polyethyleneinlage durch das Einbringen unter Vorspannung ist im Labor nicht nachweisbar [6, 8]. Tsiotikas [8] untersuchte die Einflüsse der dynamischen Belastung und ihre Auswirkungen. Vom Ergebnis her ergibt sich kein Verschleiß an der PE/ metal back Grenzfläche, das reine metal back bringt für sich alleine keine Probleme mit der Dauerfestigkeit unter normativer Belastung 
(dynamisch), weil auftretende Lasten auf solch niedrigem Niveau zu verzeichnen sind, dass die Titanlegierung hier mit einer Dauerfestigkeit von $550 \mathrm{MPa}$ weit im unterkritischen Bereich liegt.

Da die elastische Deformation des knöchernen Acetabulums einmal von der Größe des Implantates, darüber hinaus natürlich auch von seiner Steifigkeit und schließlich auch auf der knöchernen Seite von der Verformung des Knochens bei Lasteinfluss und der Qualität des Knochens abhängig ist, werden Relativbewegungen kleiner, wenn alle Knochendefekte mit patienteneigenem Knochenmaterial rekonstruiert werden und das sphärische Knochenlager nicht durch eine Ausdehnung des Fräsvorgangs sondern durch knöcherne Aufbauplastiken geschaffen wird. Die Technik führt zum Einsatz kleinerer Pfannenkomponenten. Gleichzeitig resultieren aber knöcherne Acetabula mit einer größeren Wandstärke und damit einer höheren Verformungsstabilität, und es ergibt sich automatisch eine stärkere Annäherung der Verformung des Knochens an die des Implantates [9, 10, 11].

Andererseits weist auch das natürliche Acetabulum eine subchondrale Versteifungszone in Form der Sourcil auf, die die angrenzende spongiöse Füllung des acetabulären Daches vor punktförmigen Überlastungen, welche die strukturelle Qualität der spongiösen Innenfüllung mechanisch überfordern würden, schützt und die Lasten großflächig verteilt. Dieses System soll das craniale Schalenviertel der Pfanne imitieren, indem es starr konstruiert ist. Eine zunehmende Elastizität wird durch die Abnahme der Schichtdicke und die spangenartige Öffnung der caudalen Zirkumferenz der Pfanne gewährleistet. Diese konstruktive Form kommt offensichtlich der verstärkten Verformungsmöglichkeit der ventralen und dorsalen Facies lunata entgegen und fängt auch Spitzenbelastungen des Implantat-Knochen-Verbundes auf, was unsere Messungen belegen. Zeiler [11] konnte zeigen, dass auch die caudalen Spangen des Implantates knöchern integrierten, in diesem Pfannenbereich keinerlei radiologische Aufhellungslinien zu verzeichnen waren.

Die biomechanischen Messungen an der anisotropen Vektorpfanne ergaben ein anisotropes Elastizitätsverhalten mit einer Federrate c, die im Bereich des Spangenursprungs um mehr als den Faktor 10 höher ist als im Bereich der Spangenenden. Dies entspricht einer hohen Steifigkeit des Implantates cranial im Größenbereich von $1000 \mathrm{~N} / \mathrm{mm}$ und einer entsprechend dem physiologischen Verformungsverhalten der natürlichen Hüftpfanne im caudalen Bereich angepassten erhöhten Elastizität im Größenbereich von 100 $\mathrm{N} / \mathrm{mm}$ im caudalen Bereich des Implantates. Die Messung am Prototyp (ohne Einsägung) konnte nachweisen, dass erst durch die Einsägung mit Erzeugung von jeweils zwei Spangen eine ca. um den Faktor 8 höhere Elastizität im caudalen Bereich erreicht wird. Es wurden 3 Messungen jeweils durchgeführt, die dabei auftretende Abweichung von $<0,5$ $\%$ führte zur Angabe der absoluten Zahlenwerte ohne Fehlerbalken.

Die ersten guten klinisch-radiologischen Ergebnisse [11] und die aktuellen Messungen belegen, dass das Konzept, das anisotrope Verformungsverhalten der natürlichen Hüftpfanne mit der geschilderten Spangenkonstruktion nachzuahmen, verwirklicht werden konnte.

\section{Abstract}

In hip endoprosthesis relative movement at the interface implant - bone occurs by different values of the stiffness of both bone and implant system. Relative movement causes bone atrophy als well as loosening of implant components in the hip joint. The Youngs' Modulus of bone varies in re-

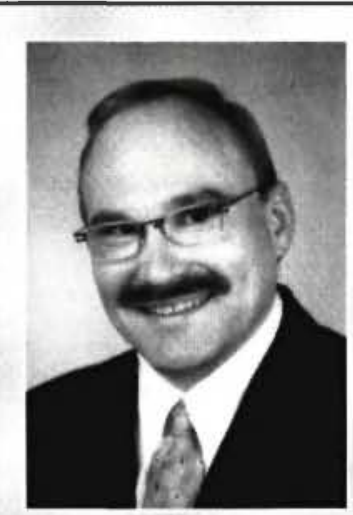

\section{Dr. med. Alexander Schuh}

Dr. med. Alexander Schuh Orthopädische Klinik Rummelsberg Rummelsberg 71 90592 Schwarzenbruck Tel. $09128 / 50-3333$ E-mail: Schuh-Alexander@ t-online.de

Akademischer Lebenslauf

1990-1995 Studium der Humanmedizin an der Friedrich-Alexander-Universität Erlangen-Nürnberg

März 1996 Promotion an der Orthopädischen Universitätsklinik Erlangen

1995-1998 Tätigkeit als AIP/ Assistenzarzt am Klinikum Weiden in der Abteilung für Unfall- und Wiederherstellungschirurgie

1998-1999 Tätigkeit als Assistenzarzt an der Orthopädischen Universitätsklinik Freiburg/Br.

Seit 1.8.1999 Tätigkeit als Assistenzarzt an der Orthopädischen Klinik Rummelsberg, Schwarzenbruck.

August 2003 Facharzt für Orthopädie

spect to age, sex and bone vitality e.g. osteoporosis. Isoelasticity never is achievable, but developing a cementless anisotropic implant design "Vektorcup" was realized by different values of stiffness changing the geometry of the cup between cranial and caudal area (anisotropic behaviour of the cup design). Aim of the present work is to prove the hypothesis that an anisotropic behaviour with this implant design was achieved.

The basic design of the anisotropic Vektorcup is a spheric shape consisting of a Titanium alloy Ti5Al2,5Fe. The metallic shell is designed with a thick wall in the cranial area, while the caudal area is designed less stiffer by using a defined flexibility by thin rips instead of closed ball shape. Checking the anisotropic behaviour of the Vektorcup we performed measurements of elasticity at several designs and prototypes of this cup at a servohydraulic test equipement, MTS 810 (MTS, Minneapolis, Minnesota). The measurements of the elasticity by determining the spring rate showed that the design concept of anisotopy was fully realized. Equatorial milling only reduced the spring rate to about $1 / 8$ of the fully shaped cup, while complete milled Vektor cup in the cranial area achieved values of $1 / 8$ comparing to the complete shell design

\section{Literatur}

[1] Bereiter H., Bürgi M., Rahn B. A.: Das zeitliche Verhalten der Verankerung einer zementfrei implantierten Hüftpfanne im Tierversuch. Orthopäde 1992, 21, 63-70.

[2] Dietschi C.: Zur Elastizität der Hüftpfanne. Swiss Med $1990,12,9-12$. 
[3] Effenberger H., Imhof M., Witzel U., Kaclin P., Rieger W.: Modification of form, material and modularity of threaded acetabulum cups. Biomed Tech (Berl). 2002, 47, 169-75.

[4] Gebauer D.: Die zementlose Hüftgelenkspfanne - Analyse der Fixierungsbedingungen der unterschiedlichen Konstruktionsprinzipien. Biomed. Technik 1987, 32, 40-45.

[5] Huggler A. H., Schreiber A., Dietschi C., Jacob H.: Experimentelle Untersuchungen über das Deformationsverhalten des Hüftazetabulums unter Belastung. Z. Orthop. 1974, 112, $44-50$.

[6] Morscher E., Bereiter H., Lampert C.: Cementless press-fit cup. Clin. Orthop. 1989, 249, 12-20.

[7] Schneider E., Schönenberger U., Giraud P. H., Bürgi M.: Primärstabilität und Beckendeformationen bei zementierten und nicht zementierten Hüftpfannen. Orthopäde 1992, 21, 57-62.

[8] Tsiotikas T.: Labortechnische und klinische Untersuchungen zum Prothesensystem Vektor. Dissertation. Erlangen, 2001 .

[9] Zeiler G., Thull R.: Klinische Voraussetzungen, mechanische Untersuchungen und erste Implantationserfahrungen mit einem flexiblen Prothesensystem für das Hüftgelenk. Biomed. Technik 1991, 36 (Ergänzungsband), 202.

[10] Zeiler G., Thull R.: Die Verankerung eines sphärischen, anisotrop-elastischen Pfannenimplantates mit Knochentransplantationen. Orthop. Praxis 1992, 28, 279-282.

[11] Zeiler G., Karan M., Holzwarth U., Schuh A. Die zementfreie anisotrope Pfanne "Vektor" in der Endoprothetik des Hüftgelenkes. Vortrag anläßlich der 27. Jahrestagung der Österreichischen Gesellschaft für Orthopädie und Orthopädische Chirurgie in Graz 2003. 